\title{
ATLAS jet measurements, and subjet structure for boosted hadronic objects
}

\section{Bertrand Chapleau*}

On behalf of the ATLAS Collaboration

McGill University

E-mail: bertrand.chapleau@cern.ch

\begin{abstract}
Comprehensive jet cross section measurements are presented using the data samples recorded in 2010 and 2011 at $\sqrt{s}=7 \mathrm{TeV}$ centre-of-mass energy by the ATLAS detector at the LHC. In addition, measurements of different event shapes constructed from jets are presented, as well as measurements probing the internal structure of jets and the presence of heavy flavour hadrons in jets.
\end{abstract}

36th International Conference on High Energy Physics

4-11 July 2012

Melbourne, Australia

\footnotetext{
* Speaker.
} 


\section{Introduction}

Jet production at the LHC is the dominant high transverse-momentum process and provides an important testing ground for our understanding of perturbative QCD in a new kinematic regime. On the other hand, hard QCD processes are a background for many new physics models that include boosted hadronically decaying objects, that is when the decay products are close to each other in the detector as a result of a large Lorentz boost and cannot be resolved by individual jets. Thanks to the fine granularity and high energy resolution of the ATLAS [1] calorimeters, the internal structure of jets thus provides crucial discrimination against backgrounds for many exotics searches involving such boosted massive particles.

\section{Jet Reconstruction}

The most commonly used jet algorithm in ATLAS is anti- $k_{t}$ [2] with radius parameters of $R=0.4$ and $R=0.6$. When dealing with boosted objects, a much larger radius parameter (up to $R=1.2$, so called large- $R$ jets or fat jets in the literature) is typically used in order to enhance the acceptance. The inputs to those jet algorithms are three-dimensional noise suppressed clusters [3] of calorimeter cells.

Jet calibration restores the measured jet energy to the particle-level jet energy scale (JES), on average. It is the main source of experimental uncertainty for many measurements. The JES uncertainty is well within $5 \%$ for central jets over a wide range of transverse-momentum $\left(p_{\mathrm{T}}\right)$ as estimated using 2010 data [4]. It is reduced even further, down to $1-2 \%$, thanks to in-situ measurements using 2011 data [5].

\section{Inclusive jet and high mass dijet cross section}

An inclusive jet double-differential cross section measurement is performed in [6] using the data sample recorded in 2010 , spanning jet $p_{\mathrm{T}}$ 's from $20 \mathrm{GeV}$ to $1.5 \mathrm{TeV}$ with a wide rapidity coverage of $|y|<4.4$. The measurement is done for both $R=0.4$ and $R=0.6$ anti- $k_{t}$ jets. As can be seen in Figure 1(a), overall good agreement is observed with NLO fixed order calculations (taking into account non perturbative corrections). It is to be noted that predictions making use of the POWHEG ${ }^{1}$ method with different parton showers (PS) Monte-Carlo (MC) do show some deviations among each other; a possible indication of some uncertainty coming from PS approximations.

Using the full 2011 data set, which amounts to $4.8 \mathrm{fb}^{-1}$, a high mass dijet double-differential cross section measurement is presented in [7], going up to a dijet system mass of 4.6 TeV. Figure 1(b) shows the good agreement with both NLOJET++ and POWHEG + PS, with the largest disagreement at very high dijet mass.

\section{Flavour composition of dijet events}

In [8], two flavour discriminating variables constructed from kinematic properties of secondary vertices found in jets were used to create MC based templates for light, charm and bottom flavour

\footnotetext{
${ }^{1}$ Details concerning all Monte-Carlo programs and event generators mentioned herein can be found in the cited document corresponding to the measurement(s) being discussed.
} 


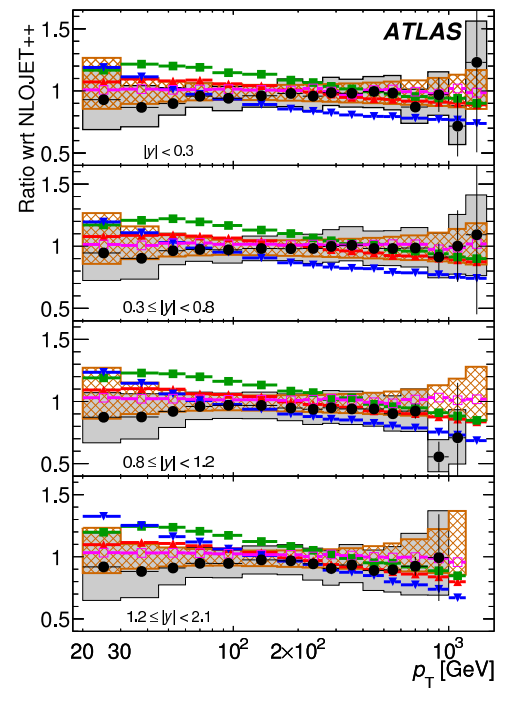

(a)

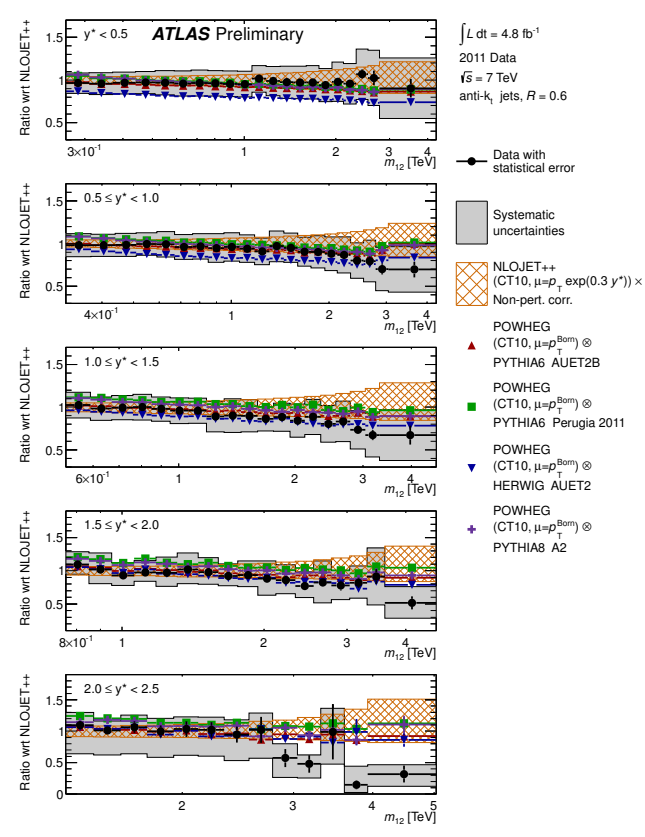

(b)

Figure 1: Ratios of the inclusive jet (a) [6] and dijet (b) [7] double-differential cross sections to the theoretical prediction obtained using $\mathrm{NLOJET}_{+}+$, as measured in the 2010 and 2011 data samples respectively. The ratios are presented in different bins of rapidity (a) and of half the absolute rapidity difference between the two leading jets (b).

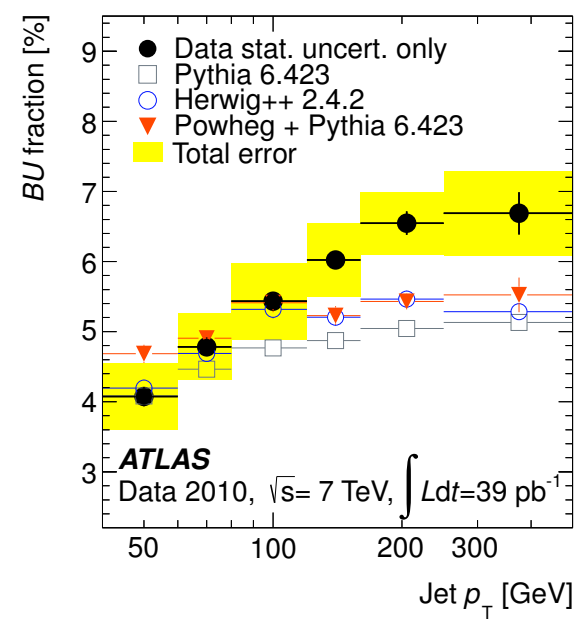

(a)

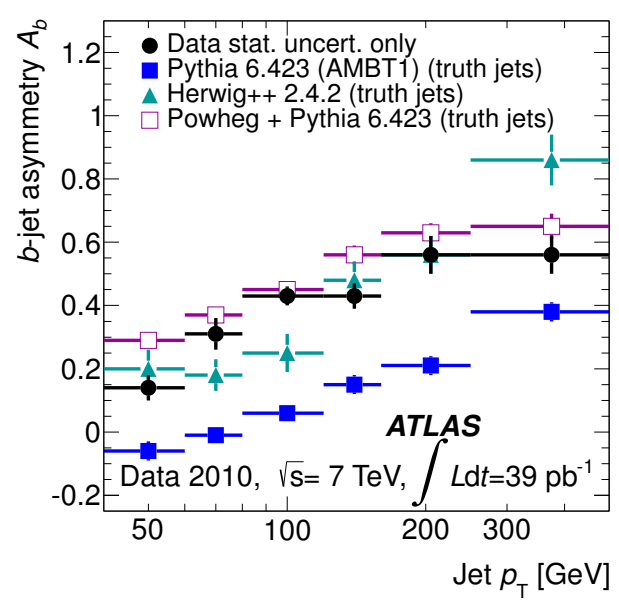

(b)

Figure 2: The unfolded dijet bottom-light flavour (that is when only one of the two leading jets contains at least one $b$-hadron) fractions for each leading jet $p_{\mathrm{T}}$ bin (a) and data fit results for the bottom dijet asymmetry (b). [8] 


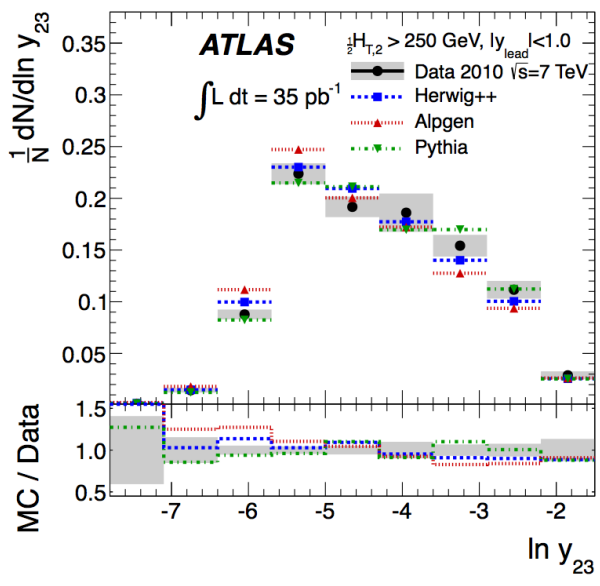

(a)

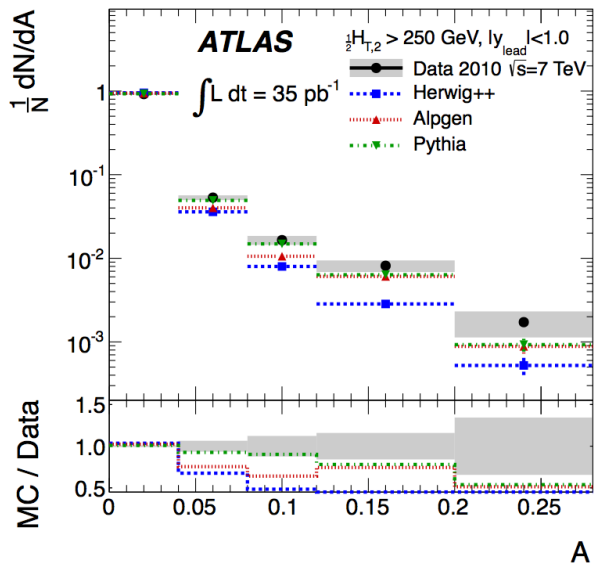

(b)

Figure 3: Unfolded hadron-level distributions of the third-jet resolution parameter, $\ln y_{23}$ (a) and aplanarity, A (b). [9]

states of jets. The flavour dijet fractions are then extracted from a multidimensional fit to the data (2010 data set). Figure 2(a) shows the bottom-light fractions for which the predictions by various $\mathrm{MC}$ generators are lower than the measured values, especially at high $p_{\mathrm{T}}$. Figure 2(b) presents the asymmetry in the number of leading and sub-leading bottom jets $\left(A_{b}=N_{b}^{S L} / N_{b}^{L}-1\right)$ which is best described by POWHEG + Pythia.

\section{Event shapes at large momentum transfer}

Event shapes characterise the event energy flow and can thus probe and test the multi-jet topologies through the use of hadronic jets. In [9], six observables were studied and fully unfolded back to particle level. Figure 3(a) shows the third jet resolution parameter $y_{23} \equiv p_{\mathrm{T}, \text { jet } 3}^{2} /\left(p_{\mathrm{T}, \text { jet } 1}+p_{\mathrm{T}, \text { jet } 2}\right)^{2}$ and Figure 3(b) the aplanarity (amount of transverse momentum with respect to the plane formed by the two hardest jets) where one can notice that Herwig++ tends to predict more planar events.

\section{Jet substructure}

The production of heavy particles with a large boost, that is with high $p_{\mathrm{T}}$, results in collimated decay products which are best captured by a single large- $\mathrm{R}$ jet. The canonical examples are boosted top quarks and boosted Higgs or $W$ bosons. QCD jets that are induced by a single light quark or gluon form an important background for searches involving boosted objects, hence the necessity to validate new jet substructure techniques.

In [10] and [11], a wide variety of such new ideas were studied with an inclusive dijet sample from the 2011 data set and compared to LO and NLO + PS predictions at the reconstructed level. Several jet grooming algorithms were investigated. Those algorithms remove components in a jet, according to a pre-defined metric and criteria, in order to better reveal the jet internal hard structure. This is an asset for large- $\mathrm{R}$ jets as it mitigates the contributions from the underlying event, pile 


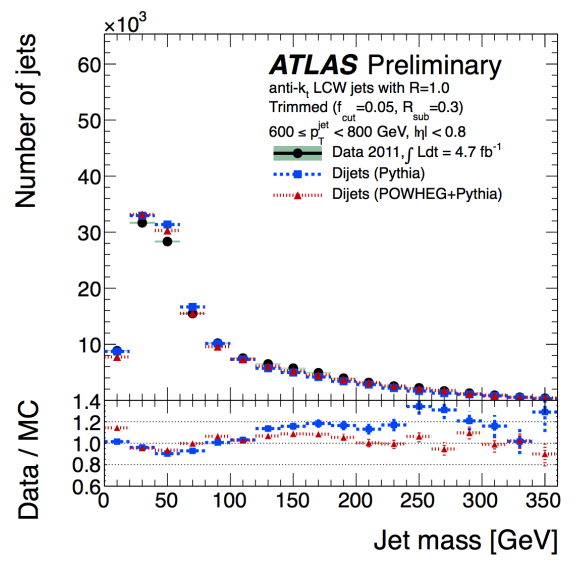

(a)

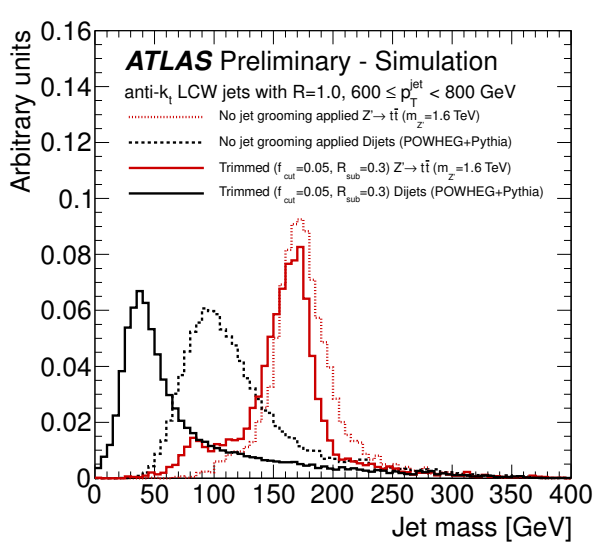

(b)

Figure 4: Jet mass for high $p_{\mathrm{T}}$ central anti- $k_{t} R=1.0$ trimmed jets (a). Jet mass for anti- $k_{t} R=1.0$ jets in simulation before and after the trimming procedure for both QCD jets and boosted top quarks (b). [10]

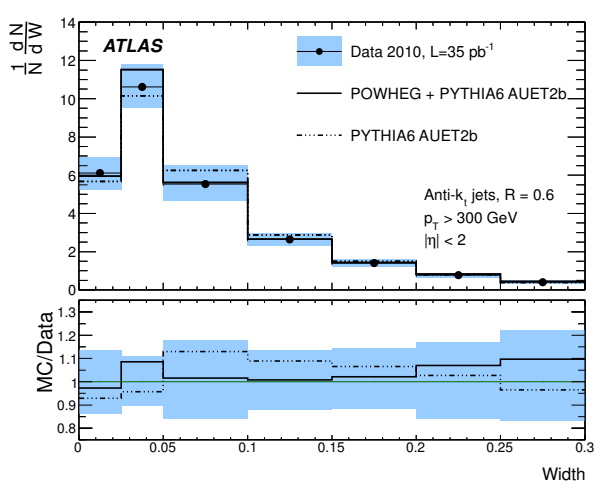

(a)

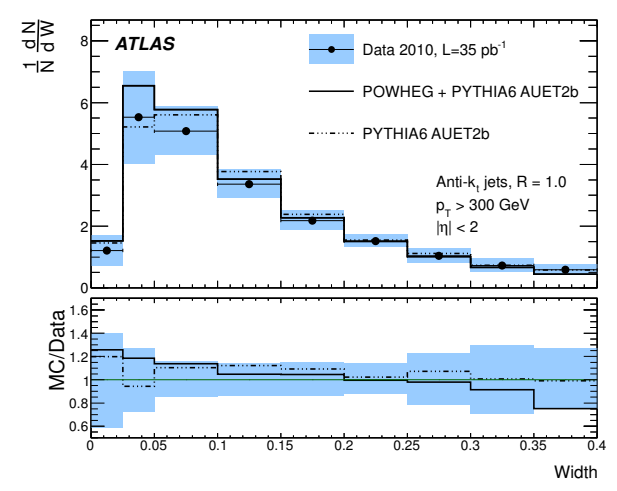

(b)

Figure 5: The jet width distributions for leading $p_{\mathrm{T}}$, anti- $k_{t} R=0.6$ jets (a) and $R=1.0$ jets (b) in the full 2010 data set, corrected for pileup and corrected to particle level. [12]

up and soft and wide-angle radiations that are not relevant to the intrinsic jet substructure. Figure 4(a) shows that there is a good description of the data by POWHEG throughout the whole range of jet masses that are within reach for this data set. A qualitative picture of the enhanced signal discrimination provided by jet grooming techniques is shown in Figure 4(b) where it is seen that the jet mass is preserved for jets with real hard substructure (as it is the case for boosted hadronic top decays) whereas it is shifted to lower values for jets with no intrinsic jet substructure (QCD jets).

Several jet shapes measurements are also presented in [12]. In that analysis, jet shapes are measured using the 2010 data set and unfolded back to the particle level for direct comparison with the theory predictions. Both standard $(R=0.6)$ and large- $\mathrm{R}(R=1.0)$ anti- $k_{t}$ jets were used with a high $p_{\mathrm{T}}$ requirement of $300 \mathrm{GeV}$. Figures $5(\mathrm{a})$ and $5(\mathrm{~b})$ show the jet width distribution $\left(p_{\mathrm{T}}\right.$ weighted 
average of the distance between the jet's constituents and its axis) for both types of jets.

\section{Conclusion}

ATLAS has performed many precision measurements to test perturbative QCD predictions in a previously unexplored kinematic regime. Results from inclusive jet and dijet double-differential cross sections, flavour composition of dijet events and event shapes at high momentum transfer were presented; overall good data and theory agreement was observed.

We are also gaining confidence in our understanding of jet substructure techniques with the ATLAS detector. Several jet substructure observables were studied in both the 2010 and 2011 data set and an exhaustive number of new jet grooming algorithms were tested which will become a key tool to enhance the discovery potential for many new physics searches in 2012 and beyond.

\section{References}

[1] ATLAS Collaboration, 2008 JINST 3 S08003.

[2] Matteo Cacciari et al, The anti-kt jet clustering algorithm, JHEP 04 (2008) 063

[3] W. Lampl et al., Calorimeter Clustering Algorithms : Description and Performance, ATL-LARG-PUB-2008-002. https : / / cdsweb. cern. ch/record/1099735

[4] ATLAS Collaboration, Jet energy measurement with the ATLAS detector in proton-proton collisions at $\sqrt{s}=7 \mathrm{TeV}$, arXiv:1112.6426

[5] ATLAS Collaboration, Probing the measurement of jet energies with the ATLAS detector using Z+jet events from proton-proton collisions at $\sqrt{s}=7 \mathrm{TeV}$, ATLAS-CONF-2012-053,

https://cdsweb.cern.ch/record/1452641

[6] ATLAS Collaboration, Measurement of inclusive jet and dijet cross sections in proton-proton collisions at 7 TeV centre-of-mass energy with the ATLAS detector, Phys.Rev. D86 (2012) 014022

[7] ATLAS Collaboration, Measurement of high mass dijet production in pp collisions at $\sqrt{s}=7 \mathrm{TeV}$ using the ATLAS detector, ATLAS-CONF-2012-021, http: //cdsweb.cern.ch/record/1430730

[8] ATLAS Collaboration, Measurement of the flavour composition of dijet events in pp collisions at $\sqrt{s}=7 \mathrm{TeV}$ with the ATLAS detector, arXiv:1210.0441

[9] ATLAS Collaboration, Measurement of event shapes at large momentum transfer with the ATLAS detector in pp collisions at $\sqrt{s}=7 \mathrm{TeV}$, arXiv:1206.2135

[10] ATLAS Collaboration, Performance of large- $R$ jets and jet substructure reconstruction with the ATLAS detector, ATLAS-CONF-2012-065, http: / / cdsweb. cern. ch/record/1459530

[11] ATLAS Collaboration, Studies of the impact and mitigation of pile-up on large-R and groomed jets in ATLAS at $\sqrt{s}=7 \mathrm{TeV}$, ATLAS-CONF-2012-066,

http: //cdsweb.cern.ch/record/1459531

[12] ATLAS Collaboration, ATLAS measurements of the properties of jets for boosted particle searches, Phys.Rev. D86 (2012) 072006 